\title{
A critical discussion on parametric and nonparametric regression methods applied to Hipparcos-FK5 residuals
}

\author{
F. J. Marco ${ }^{1}$, M. J. Martínez ${ }^{2}$, and J. A. López ${ }^{1}$ \\ ${ }^{1}$ Dept. Matematicas, Universidad Jaume I, Campus de Riu Sec, 12071 Castellon, Spain \\ e-mail: marco; lopez@mat.uji.es \\ 2 Dept. Matematica Aplicada. Universidad politecnica de Valencia ETSII Valencia, Spain \\ e-mail: mjmartin@mat.upv.es
}

Received 3 October 2003 / Accepted 22 January 2004

\begin{abstract}
In this paper we present an analysis of the differences between the Hipparcos and FK5 catalogues. We study parametric and nonparametric methods with two main objectives: first, to determine whether or not there is a pure rotation between the two catalogues, and to decide which model best represents the residuals; second, to give a practical formulation to reduce positions between the two systems at any point on the celestial sphere.
\end{abstract}

Key words. astrometry - catalogs - reference systems

\section{Introduction}

In 1991, a decision was taken by the IAU that the celestial reference system should be materialised by a celestial reference frame defined by the precise coordinates of extragalactic radio sources. The adoption of the ICRS as a new reference system was decided on the IAU assembly held in Kyoto in 1997 and it came into use in January 1998. Nevertheless, the reference frame initially related to the system, the ICRF, could not be used directly by most astronomers working in optical or infrared wavelength. Consequently, the Hipparcos catalogue (1997), with over 118300 stars, is still the optical materialization of the System today. Its positions and proper motions contain errors around 1 mas and 1 mas/yr. After the publication of Hipparcos and its acceptance as the fundamental reference frame, the question of catalogue homogenization has reemerged, following the IAU recommendation that the necessary studies should be conducted in order to obtain as thorough relationships as possible with other catalogues. In this respect, some significant papers are the ones of Mignard \& Froeschle (1997, 2000), who carried out a wide study of the deviations of the FK5 catalogue (Fricke et al. 1988) from Hipparcos; and the ones of Schwan who, in his paper (2001a) conducts a global and a zonal study of the differences between the same catalogues in the mean epoch of FK5 and following the indications of IAU (1976). In further papers Schwan (2001b, 2002), extends this work to several commonly used catalogues.

Send offprint requests to: F. J. Marco,

e-mail: marco@mat.uji.es
These two papers are concerned with two fundamental and related questions: a) the issue of whether or not of a pure rotation exists between Hipparcos and the older FK5 catalogue. Geometrical \& Linear (2001a, henceforth GL) and Geometrical and Vectorial Spherical Harmonics (henceforth GV) are used to obtain the coefficients of the geometrical infinitesimal rotations; b) the question of how a model of correction could be provided, if indeed this is possible, which would be applicable to the whole sphere. On this second question, Schwan (2001a) provides a development in spherical harmonics (modified in order to include the magnitude as a variable), while Mignard \& Froeschlé (2000), conclude that this procedure is not suitable and provide a table to interpolate. Nevertheless, all the authors coincide on the first question and conclude that the existence of a pure rotation and similar values for the infinitesimal rotations are obtained if they are computed at the same epoch. A number of crucial points related to the model used in the adjustment must be highlighted for two different reasons:

1) The first one is concerned with the importance of the consequences derived from the applications of a determined model. We identify at least two important objectives that would benefit from the correct relationships between two catalogues being obtained. The first of these is to monitor the system adequately. To this end, we only have to consider a simple relationship, such as that between GL and their temporal variations, which are related to the differences in proper motions, but not only to them: it suffices to remember the "dynamical" studies carried out into the errors in the FK5 and their temporal evolution (from 
corrections to the zero-point as in Batrakov \& Chernetenko 1997; Batrakov et al. 1999; Branham 1992; Branham \& Sanguin 1994; Shuygina \& Yagudina 1995; Vityazev \& Yagudina 1997; Yagudina 1996, 2001), and also to be applied to the masses estimation as in (Hilton 1996; Krasinsky et al. 2001; Rapaport \& Viateau 1996; Viateau \& Rapaport $1995,1997)$ or even the "tentative interpretation" given by Mignard \& Froeschlé (2000) according to a variation of the precession constant. (See also Zhu \& Yang 1999, on the same question, using the PPM and ACRS catalogues). In short, it is of interest to determine the most suitable model, given the fact that for the above-mentioned studies the use of one model does not provide the same results as the use of another. A second objective is, it may be say, static and consists of being able to relate, at any given instant, a correction to the position in one system to obtain an accurate reduction in the other. We need a function, whether explicitly given or not, that approximates the initial points with sufficient accurancy and that is also defined over the whole sphere with the least possible standard deviation. In a later section it will become evident that the standard deviations obtained from the use of rotations plus spherical developments will render them unsuitable for this purpose.

2) The second motivation is of mathematical nature concerning the methods, models and their expected properties. This conditions the way rest of the paper is organised. Specifically, we show that the selection of the correction model commonly used, the GL model, is not suitable for use bias exists in the data. We propose the use of an unbiased model since this is the only model that provides minimum variance when applying the least square method. We also show that this implies a considerable change in the value of the rotation around the $z$-axis. These results are confirmed by means of two independent verifications using spherical harmonics and regression kernels, respectively. Finally, we conclude, with Mignard \& Froeschle (1997) that it is not appropiate to apply an analytical expression to corrections on the whole sphere and it is possible to provide a table resulting from a nonparametric regression method which strongly agrees when it is only applied to the basis points.

In order to clarify all the questions that arise from the problems we are concerned with, we have included a section devoted to a critical discussion about the correct use of the usual mathematical background as it is related to three specific models of correction. In further sections, we consider the selection of the data and their properties, the GL and GV models with a critical comment that will lead us to the introduction of the GLAD model (GL model plus bias coefficients to the right ascension and declination). Furthermore, we use kernel regression and spherical harmonics adjustment to contrast the values for the most simple models directly obtained. This is a good property that assures the internal coherence of the results obtained. The paper ends with two final sections corresponding to the two main objectives, which are outlined in the preceding paragraphs. The first of these provides two independent verifications of the accuracy of order of the magnitude of our $\epsilon_{z}$ value from the GLAD model, which is independent, and this is a very important point, of the initial model adopted: regardless of wether it is GL or GLAD. The second concerns the use of mixed models for the global adjustment to be applied to the whole sphere and we work only with parametric (GL or GLAD with spherical harmonics adjustment) or GL or GLAD plus non-parametric models (hence, the mixed name used). In this section the use of a GL or GLAD model as a basis model is not important if we only examine the final results, but this is not the case if we also look at the contribution of both components in the result.

\section{A critical revision of mathematical models and commonly used methods in the analysis of a discrete set of differences on the sphere}

\subsection{The models}

Over recent decades, a large number of papers dealing with the relationships among different catalogues have been published. The aim of all these papers is to obtain functional relationships among catalogues to approximate the differences $\Delta \alpha \cos \delta$ and $\Delta \delta$. They are obtained by employing different models on a discrete set of points. Of the different models dealing only with the complete sphere, we can highlight those that obtain a transformation by means of infinitesimal rotations, those that pursue harmonical developments for each one of the random variables separately (Schwan 2001a; Bien et al. 1978), those that, from spherical harmonics developments for each variable attemp to deduce through the use of statistical methods, whether or not a transformation by infinitesimal rotations exists (Vityazev 1997a) and also those that look for vectorial spherical harmonics developments (Mignard \& Froeschlé 1997, 2000). Below, we strongly contend that different questions must be taken into account in order to analyse the reliability of the results obtained. In particular, there are several mathematical disciplines clearly attached to the building of models and to the computation of parameters and several properties dealing with those disciplines that play a part in this reliability. We specify them in the following points:

1a) The surface spherical harmonics developments (henceforth $\mathrm{SH}$ ) are based on the hypothesis that the developed functions have an integrable square on the sphere. In this case, the coefficients are found according to precise formulas in a simple way due to the functional orthogonality of these harmonics. Also, this orthogonality implies that if the truncated series of increasing orders is computed, to better approximate the original function, the coefficients may be used for the next orders. It is interesting to note that the computation of the coefficients $\left\{c_{j}, j \geq 0\right\}$, for a given function $f$, and where a truncation order $n$ is $\left\{Y_{j}, j \geq 0\right\}$, verify the property of minimizing the integral:

$\int_{S^{2}}\left[f(\alpha, \delta)-\sum_{j=0}^{n} c_{j} Y_{j}(\alpha, \delta)\right]^{2} \mathrm{~d} \sigma$

where $\mathrm{d} \sigma$ is the area element in the spherical domain. 
1b) In general, a vectorial field may be linearly approximated around the identity by means of the addition of an antisymmetric and a symmetrical transformation. If the field is given by the difference $X(\alpha+\Delta \alpha, \delta+\Delta \delta)-X(\alpha, \delta)$ where $X$ is the unitary tridimensional vector in spherical coordinates, the antisymmetric part is geometrically related with a transformation by means of infinitesimal rotations and the symmetrical part is related with a possible deformation that, strictly speaking, makes no sense. Thus, we have the expression

$$
\frac{1}{\cos \delta} \frac{\partial X}{\partial \alpha}(\Delta \alpha \cos \delta)+\frac{\partial X}{\partial \delta}(\Delta \delta)=A X
$$

where

$$
A=\left[\begin{array}{ccc}
0 & \epsilon_{z} & -\epsilon_{y} \\
-\epsilon_{z} & 0 & \epsilon_{x} \\
\epsilon_{y} & -\epsilon_{x} & 0
\end{array}\right]
$$

Orthogonality of vectors $X, \frac{1}{\cos \delta} \frac{\partial X}{\partial \alpha}, \frac{\partial X}{\partial \delta}$ with respect to the usual scalar product leads to the fact that the following relationships should be accomplished in order to obtain the necessary and sufficient conditions for the existence of (2):

$(\Delta \alpha) \cos \delta=-\epsilon_{x} \cos \alpha \sin \delta-\epsilon_{y} \sin \alpha \sin \delta+\epsilon_{z} \cos \delta$

$\Delta \delta=\epsilon_{x} \sin \alpha-\epsilon_{y} \cos \alpha$

1c) Still more generally, we can consider the vector field on the unitary sphere, given by $[(\Delta \alpha) \cos \delta, \Delta \delta]^{t}$. We know that under certain regularity hypothesis it has a development in vectorial spherical harmonical functions given by (5), (Morse \& Feshbach 1953)

$R_{n, m}=\frac{\vec{r}}{r} Y_{n, m}, S_{n, m}=r \overrightarrow{\nabla Y}_{n, m}, T_{n, m}=-\vec{r} \times \overrightarrow{\nabla Y}_{n, m}$

where $Y_{n, m}(n \geq 0,-n \leq m \leq m)$ are the usual spherical harmonics. If we consider the truncated development:

$\overrightarrow{\Delta X}=\sum_{j=-1}^{1}\left[c_{1, j} \vec{R}_{1, j}+d_{1, j} \vec{S}_{1, j}+e_{1, j} \vec{T}_{1, j}\right]$

where $\overrightarrow{\Delta X}=\vec{X}(r+\Delta r, \alpha+\Delta \alpha, \delta+\Delta \delta)-\vec{X}(r, \alpha, \delta)$ is approximated in first order, the compatibility of the system must be accomplished, which implies the functional relationships:

$(\Delta \alpha) \cos \delta=\left[e_{1,0} \cos \delta-e_{1,1} \sin \alpha \sin \delta-e_{1,-1} \cos \alpha \sin \delta\right]$

$+\left[d_{1,1} \cos \alpha-d_{1,-1} \sin \alpha\right]$

$\Delta \delta=\left[e_{1,-1} \sin \alpha-e_{1,1} \cos \alpha\right]$

$+\left[-d_{1,1} \sin \alpha \sin \delta-d_{1,-1} \cos \alpha \sin \delta+d_{1,0} \cos \delta\right]$

where it is evident that $e_{1,-1}=\epsilon_{x}, e_{1,1}=\epsilon_{y}, e_{1,0}=\epsilon_{z}$ with $\epsilon_{x}, \epsilon_{y}$ and $\epsilon_{z}$ are infinitesimal rotations around the corresponding axis. Hence, this model generalizes the previous one.

\subsection{The methods and their compatibility with the various models}

If we denote any of the second members of the models given in 1a), 1b) and 1c) as $\operatorname{Model}(\alpha)$ and $\operatorname{Model}(\delta)$, it is also possible, a priori, to compute the coefficients that minimize the integral of the residuals:

$\int_{S^{2}}\left\{[(\Delta \alpha) \cos \delta-\operatorname{Model}(\alpha)]^{2}+[\Delta \delta-\operatorname{Model}(\delta)]^{2}\right\} \mathrm{d} \sigma$

The difference between SH, GV in one way and GL in the other way is evident: the condition of the minimization in the $L^{2}\left(S^{2}\right)$ norm to compute the coefficients of the model is inherent to the fact that the function ( $\mathrm{SH}$ case) or the vectorial field (GV case), under certain regularity hypothesis, may be developed in a series (of spherical surface and vectorial harmonics respectively). In both cases we are projecting a function on the functional subspaces generated by all the functions with orders equal or lower than the order of the development pursued. In both methods it is possible to increment the order arbitrarily (at least, theoretically. This is not so if we have a discrete set of points, as in our case). In the GL method the order is fixed and, in functional terms, it does not reach first order, because GV is the only one of first order.

In addition, when we have a finite number of points we must use special methods to obtain solutions close to the theoretical ones. These methods may be "grosso modo" classified in two kinds, the discretization methods and the statistical regression methods, in particular the parametric ones. In the first type we work with functions, while in the second, we work with random variables. In this vein, according to the desirable properties in each one of the procedures, the following remarks can be made:

2a) The spatial distribution of the points over the sphere. When the distribution in the $\alpha$ and $\sin \delta$ variables is uniform (with discretization of differentials such as $\mathrm{d} \alpha \cos \delta \mathrm{d} \delta=$ $\mathrm{d} \alpha \mathrm{d}(\sin \delta) \approx h k$ with $h, k$ constants $)$, the discretization of the integral using the method of rectangles provides the same summation as that which appears statistically, if a model originating from the truncation of a series of orthogonal functions is used (in surface spherical harmonics or in vectorial spherical harmonics). Small variations in this property will give small variations in the results, and the property given in the next point must be taken into account.

2b) The application of a parametric regression statistical method is based on the Gauss-Markov theorem, which affirms that the minimum-quadratic estimator is the best of the ones that appear when the residuals are normally distributed with null mean and variance $\sigma^{2}$. Thus, if the random variable is not normal with null mean, the least squares method cannot give us the unbiased estimator of minimum variance that we are looking for.

The difference between $2 \mathrm{a}$ and $2 \mathrm{~b}$ should be noted. The former refers to the fact that the spatial distribution of the points $\left(\alpha_{i}, \delta_{i}\right)$ on the celestial sphere is, more or less, homogeneous. The latter refers to the distribution of the random variables $\Delta \alpha \cos \delta$ and $\Delta \delta$. It is clear that the effectiveness 
of the selected model depends on both properties and the absence of either of them may be determinant, fundamentally with the 2 b property, as we shall see in the cases we are dealing with in the present paper.

It should finally be pointed out that, in practice, we are looking for a functional adjustment (with its previously signalled differences and the importance for the expected results), by means of a least squares method (When using this method the corresponding properties must also be observed). The stability of the results, a concept that must be revised for each case, will give us qualitative information about the solution obtained, and will thus be an index of its reliability. Below, we continue with the data selection and the study of their properties, in the way already mentioned in $2 \mathrm{a}$ and $2 \mathrm{~b}$.

\section{Choice and previous study of the data set}

To carry out the present study we considered the catalogues Hipparcos (ESA 1997) and FK5 (Fricke et al. 1988). We agree with Schwan in that from a methodological point of view, it is appropiate to make the comparison between the two catalogues at the $\mathbf{J} 2000$ epoch since the proper motions from the Hipparcos catalogue are better than those from the FK5 catalogue (Schwan 2001a). However, our study is undertaken at 1991.25 for two reasons. The first of these is of a practical nature, because the "official" relationships given by infinitesimal rotations between the two catalogues, which are included in Vol 3 of Hipparcos, established by Mignard and Froeschlé, and the further study published by the same authors (Mignard \& Froeschlé 2000) are made at 1991.25 The second reason is that Schwan himself, in his previously mentioned paper, concludes that the transfer of the results that he obtains from J2000 to 1991.25 give very similar results to these. Thus, our reference date will be 1991.25. In the first step, we applied the corresponding proper motions to the FK5 stars. Then, we selected the star according to the same criterion used by Schwan (2001a), so 1327 stars verifying the conditions (10) were selected for use in the studies presented throughout the paper:

$$
\begin{aligned}
& \sqrt{(\Delta \alpha \cos \delta)^{2}+(\Delta \delta)^{2}} \leq 10^{-5} \mathrm{rad} \\
& \sqrt{\left(\Delta \mu_{\alpha} \cos \delta\right)^{2}+\left(\Delta \mu_{\delta}\right)^{2}} \leq 10 \text { mas. }
\end{aligned}
$$

The characteristics of the data that we are particularly interested in are as follows: firstly, the spatial distribution of the stars in order to see how close they come to forming an homogeneous sample; secondly, the statistical distribution of the random variables $\Delta \alpha \cos \delta$ and $\Delta \delta$, considered as a set of numerical data. The spatial distribution may be seen in Fig. 1 where $\alpha$ is found in the abscise axis, $\sin \delta$ in the ordinates axis and the distribution, which is practically homogeneous, was approximated by means of the Epanechnikov kernel (Simonoff 1996) for two variables (see Formula (12)).

From a numerical point of view, we computed the arithmetical mean and the standard deviation of the corresponding variables and also, by means of the Epanechnikov kernel, we computed the expectation and the standard deviation from the

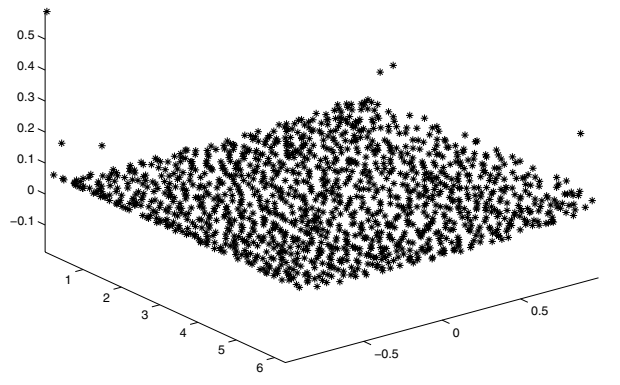

Fig. 1. Spatial distribution of the data.

Table 1. Means and standard deviations (results in mas).

\begin{tabular}{lllll}
\hline \hline & $\mu$ & $\sigma$ & $E[]$ & $\sigma$ \\
\hline$\Delta \alpha \cos \delta$ & $11^{\prime} 41$ & $83^{\prime} 46$ & $11^{\prime} 43$ & $88^{\prime} 15$ \\
$\Delta \delta$ & $-46^{\prime} 03$ & $89^{\prime} 16$ & $-45^{\prime} 75$ & $96^{\prime} 60$ \\
\hline
\end{tabular}

expectation of the variables, of their squares, using the wellknown formulas (11):

$\mu=E[X]=\int_{D} x f_{X}(x) \mathrm{d} x \quad \sigma^{2}=\operatorname{Var}(X)=E\left[X^{2}\right]-(E[X])^{2}$

where

$f_{X}(x)=\frac{1}{h} \sum_{i=1}^{n} K\left(\frac{x-x_{i}}{h}\right), K(x)=\frac{3}{4}\left(1-x^{2}\right)$

where $K(x)$ is the Epanechnikov kernel (Simonoff 1996). Due to the discrete character of this computation (the function approached by means of a kernel regression has no exact expression) the integrals were approximated using the method of rectangles and the trapezoidal rule. (In both cases the same values were obtained). The results obtained may be seen in Table 1 .

Finally, the dependence of the two random variables is easily verified as the joint density function and the product of the two individual variables differ significantly.

To summarize this paragraph, it shuold be noted that the random variables $\Delta \alpha \cos \delta$ and $\Delta \delta$ are not independent, and approximately distribute as normal random variables (Figs. 2-4) with mean not null (whose values, together with the corresponding standard deviations are given in Table 1) and that they are spatially distributed over the sphere in a sufficiently homogeneous way (Fig. 1).

\section{Infinitesimal rotations, vectorial harmonics models and statistical properties of the residuals}

Once a dependence between the random variables had been established through experiment, it made sense to look for a model of adjustment where parameters common to both of them appear. As we explained in the introduction, the most frequently used model considers that there is a transformation by infinitesimal rotations between the two catalogues. This model leads to Eqs. (3) and (4) has also been used recently. The model deduced from the development of the vectorial field of the residuals in right ascension and declination by means of vectorial spherical harmonics (Eqs. (7) and (8)). 


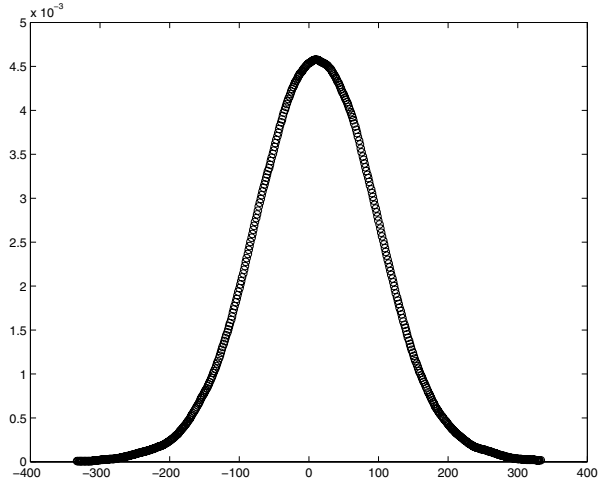

Fig. 2. Density function for $\Delta \alpha \cos \delta$ (we used the Epanechnikov kernel, $h=66$ mas).

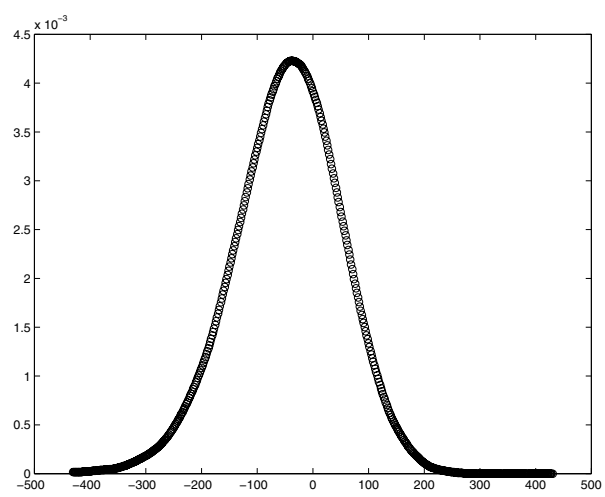

Fig. 3. Density function for $\Delta \delta$ (we used the Epanechnikov kernel, $h=86$ mas)

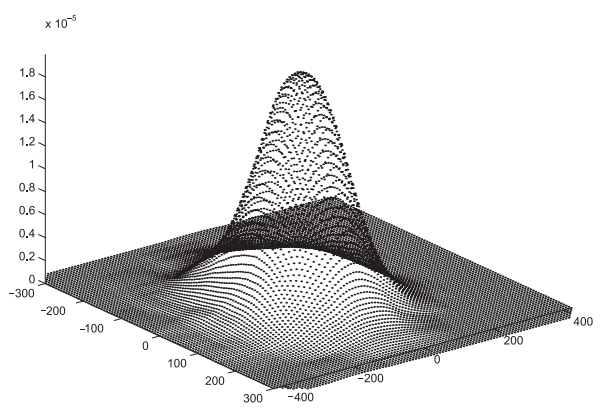

Fig. 4. Joint distribution for $\Delta \alpha \cos \delta$ and $\Delta \delta$ (we used the Epanechnikov kernel, $h_{\alpha}=66$ mas, $h_{\delta}=86$ mas).

Table 2 reflects the results we obtained with the GL and GV compared with those of (Mignard \& Froeschle 2000, henceforth MF). In numerical terms, the coefficient values are very similar for the GL coefficients, the numerical differences depending on the basis set of stars selected. The small differences given by MF in (1997) and (2000) can also be observed: they obtain $\epsilon_{x}=-18.8$ and $-17.3, \epsilon_{y}=-12.3$ and -14.3 , $\epsilon_{z}=16.8$ and 16.8 mas. In spite of the fact that the difference between the methods employed by MF and Schwan is also found in the study of the zonal residuals (this pointis dealt with below), in the context of this section we only consider that the pure rotations relating the two catalogues are computed
Table 2. Results from MF and our results (GL and GV) (Results in mas).

\begin{tabular}{lllllll}
\hline \hline & $\epsilon_{x}$ & $\epsilon_{y}$ & $\epsilon_{z}$ & $d_{1,0}$ & $d_{1,1}$ & $d_{1,-1}$ \\
\hline $\mathrm{GL}$ & -18.4 & -14.8 & 18.7 & - & - & - \\
$\mathrm{GV}$ & -21.4 & -18.6 & 20.4 & -60.1 & 0.9 & 25.7 \\
$\mathrm{MF}$ & -17.3 & -14.3 & 16.8 & - & - & - \\
\hline
\end{tabular}

Table 3. Means of various models compared with the mean of the sample (in mas).

\begin{tabular}{lcccccc}
\hline \hline & Initial $\mu$ & GL & GV without def & GV with def & MF & GLD \\
\hline$\Delta \alpha \cos \delta$ & 11.4 & 15.0 & 14.8 & 14.9 & 13.5 & 15.0 \\
$\Delta \delta$ & -46.0 & 0.5 & 0.6 & -46.8 & 0.5 & -46.0 \\
\hline
\end{tabular}

from different models, as Schwan uses the GL model (Eqs. (3) and (4)) and MF use the GV model (Eq. (5)).

The values for the deformation elements of MF were not available and thus they do not appear here. It is noticeable that, with the exception of $d_{1,1}$, they cannot be neglected, but we presume that they were not published because the zonal study must absorb these differences. In all events, from a purely statistical point of view, we are interested in results that allow us to study the reliability of the adjustment. In the first place, the means (expectation) of the random variables and those from the second members should be coincident if the model is good. This would indicate that we have an unbiased model, which would lead to the fact that the application of the Gauss-Markov theorem will assure us that, in the clategory of the functional estimators considered (which are, in fact, determined by the parameters), the one obtained is that of the least variance. The kernel method can be used to check whether the property is verified, but, for our purposes, it is sufficient to compute the mean of both members on the set of 1327 selected common stars. The "a priori" means of the differences $\Delta \alpha \cos \delta$ and $\Delta \delta$ are, respectively 11.4 and -46.0 mas. The second members (estimators) provide the means listed in Table 3.

In conclusion, for the random variable $\Delta \alpha \cos \delta$ the mean is not reached with any of the models. For $\Delta \delta$ all the models are biased, with the exception of the GV, when the deformation terms are also considered. To maintain the most simple possible unbiased model for both random variables, we can modify the GL by adding a constant $\Delta D$ in formula (4), (GLD Model, Lopez et al. 1993). After making the adjustment the values $\Delta D=-46.6, \epsilon_{x}=-19^{\prime} 1, \epsilon_{y}=-16^{\prime} 3$ and $\epsilon_{z}=18^{\prime} 6$ are obtained, providing the means for the adjustments that appear in Table 3. In conclusion, we can see that the bias in declination has been absorbed in the GLD model, but not the bias in $\Delta \alpha \cos \delta$.

\section{The unbiased GLAD model}

Following the last section, our aim is to obtain a unbiased modified GL model for both random variables. For this reason we include a $\Delta A$ in the second member of (3), as we previously 
Table 4. Parameter values for the GLAD, MF, GL and GLD methods and means and standard deviations for the residuals (in mas).

\begin{tabular}{llllllllll}
\hline \hline & $\epsilon_{x}$ & $\epsilon_{y}$ & $\epsilon_{z}$ & $\Delta A$ & $\Delta D$ & $\mu\left(\right.$ res $\left._{\alpha}\right)$ & $\mu\left(\right.$ res $\left._{\delta}\right)$ & $\sigma\left(\right.$ res $\left._{\alpha}\right)$ & $\sigma\left(\right.$ res $\left._{\delta}\right)$ \\
\hline GLAD & -19.1 & -15.6 & 84.7 & -56.2 & -46.0 & 0.0 & 0.0 & 82.41 & 85.99 \\
MF & -17.3 & -14.3 & 16.8 & - & - & -2.1 & -45.6 & 83.48 & 86.14 \\
GL & -18.4 & -14.8 & 18.7 & - & - & -3.6 & -45.6 & 83.48 & 86.06 \\
GLD & -19.1 & -16.3 & 18.6 & - & -46.6 & -3.5 & 0.0 & 83.58 & 85.96 \\
\hline
\end{tabular}

did to eliminate the bias in declination. This model is denoted GLAD (see (13) and (14)).

It may be observed that the GLAD method absorbs the bias for the right ascension and for the declination. This is explainable in terms of the unbiased properties and of the properties of least variance: indeed, if we take the generic model:

$(\Delta \alpha) \cos \delta=\Delta A-\epsilon_{x} \cos \alpha \sin \delta-\epsilon_{y} \sin \alpha \sin \delta+\epsilon_{z} \cos \delta$

$\Delta \delta=\Delta D+\epsilon_{x} \sin \alpha-\epsilon_{y} \cos \alpha$

and we compute the values of the parameters in such a way that they minimize

$\left[\frac{1}{N} \sum_{i=1}^{N} \Phi\left(\alpha_{i}, \delta_{i}, \epsilon_{x}, \epsilon_{y}, \epsilon_{z}\right)-\overline{\Phi\left(\alpha, \delta, \epsilon_{x}, \epsilon_{y}, \epsilon_{z}\right)}\right]^{2}$
$+\left[\frac{1}{N} \sum_{i=1}^{N} \Psi\left(\alpha_{i}, \delta_{i}, \epsilon_{x}, \epsilon_{y}, \epsilon_{z}\right)-\overline{\Psi\left(\alpha, \delta, \epsilon_{x}, \epsilon_{y}, \epsilon_{z}\right)}\right]^{2}$

with $N=1327$ representing the number of stars and the line over the functions denoting arithmetical mean. The results obtained are from GLAD, listed in Table 4. It should be noted that in order to apply the normal equations, the means of products such as $\cos \alpha \sin \delta$, etc. must be previously computed. The functions $\Phi$ and $\Psi$ are:

$$
\begin{aligned}
\Phi\left(\alpha, \delta, \epsilon_{x}, \epsilon_{y}, \epsilon_{z}\right)= & (\Delta \alpha) \cos \delta-\left(-\epsilon_{x} \cos \alpha \sin \delta\right. \\
& \left.-\epsilon_{y} \sin \alpha \sin \delta+\epsilon_{z} \cos \delta\right) \\
\Psi\left(\alpha, \delta, \epsilon_{x}, \epsilon_{y}, \epsilon_{z}\right)= & \Delta \delta-\left(\epsilon_{x} \sin \alpha-\epsilon_{y} \cos \alpha\right) .
\end{aligned}
$$

It may be observed that the initial bias has been correctly absorbed, but the values of $\epsilon_{z}$ are quite different from those obtained by other methods that only use infinitesimal rotations.

While the GLAD method absorbs the bias in Right Ascension and in Declination, the other methods show a good behaviour in Declination but not in Right Ascension, which may be explained by the fact that these models contain the term $\epsilon_{z} \cos \delta$. In spite of the fact that the GLAD model is the one that best agrees with the unbiased condition, the variation in the coefficient of the $\epsilon_{z}$ parameter requires an explanation for it to be accepted or rejected. This question will be studied below.

\section{Model contrast using kernel regression}

It should be remembered that nonparametric adjustments by kernels compute the conditional mean of a certain random variable that depends on other variables. Thus, for example, if $X$ is the random variable $(\Delta \alpha \cos \delta$ or $\Delta \delta)$ the method consists of finding

$$
\begin{aligned}
m_{X}(\alpha, \delta) & =E[X \mid(\alpha, \delta)]=\int_{D} x f(x \mid \alpha, \delta) \mathrm{d} x \\
& =\int_{D} x \frac{f(x, \alpha, \delta)}{f_{(\alpha, \delta)}(\alpha, \delta)} \mathrm{d} x
\end{aligned}
$$

where $D$ is the spherical domain of $X, f(x, \alpha, \delta)$ is the joint density function of the three variables and $f_{(\alpha, \delta)}(\alpha, \delta)$ is the marginal density (Wand \& Jones 1995). Of course, all of them may be unknown so they should be approximated in some way and, to this end, we can proceed in the same way as in the previous section. Thus, we approximate

$$
\begin{aligned}
\widehat{f}(x, \alpha, \delta)= & \frac{1}{n h_{x} h_{\alpha} h_{\sin \delta}} \sum_{i=1}^{n} K_{x}\left(\frac{x-x_{i}}{h_{x}}\right) \\
& \times K_{\alpha}\left(\frac{\alpha-\alpha_{i}}{h_{\alpha}}\right) K_{\delta}\left(\frac{\sin \delta-\sin \delta_{i}}{h_{\sin \delta}}\right)
\end{aligned}
$$

and the condition

$\frac{1}{4 \pi \mu(D)} \int_{D} \int_{S^{2}} \widehat{f}(x, \alpha, \delta) \cos \delta \mathrm{d} x \mathrm{~d} \alpha \mathrm{d} \delta=1$

must be fulfilled. The same occurs with the marginal density. Selecting the same kernel and taking into account its properties we arrive at an expression similar to the Nadaraya-Watson expression, but in the sphere:

$m_{X}(\alpha, \delta)=\sum_{i=1}^{n} \omega_{i} x_{i}, \omega_{i}=\frac{K\left(\frac{\alpha-\alpha_{i}}{h_{\alpha}}\right) K_{\delta}\left(\frac{\sin \delta-\sin \delta_{i}}{h_{\sin \delta}}\right)}{\sum_{j=1}^{n} K_{\alpha}\left(\frac{\alpha-\alpha_{j}}{h_{\alpha}}\right) K_{\delta}\left(\frac{\sin \delta-\sin \delta_{j}}{h_{\sin \delta}}\right)}$.

This method has the following property: if we take $h_{\alpha} \rightarrow 0$ and $h_{\sin \delta} \rightarrow 0$ then $m\left(\alpha_{i}, \delta_{i}\right) \rightarrow x_{i}$. In other words, the lower $h$ is, the more abrupt the adjustment and, on the contrary, a large $h$ gives a smoother result. The theoretical optimum values are $h_{\alpha}=0.55, h_{\sin \delta}=0.17$ which were deduced from the expression $H=\left(\frac{4}{d+2}\right)^{\frac{1}{d+4}} \Sigma^{\frac{-1}{2}} n^{\frac{-1}{d+4}}$ (Simonoff 1996), where $H$ is the vector of the different values of $h, d=2$ (dimension), $n$ the number of points and $\Sigma$ the variance-covariance matrix of the random variables of the joint spatial distribution for $(\alpha, \sin \delta)$ that, as previously mentioned, has two practically uniform random and, of course, independent variables. Despite this expression being true when a Gauss kernel is used, we experimentally checked that the adjustment is similar to that one obtained with the Epanechnikov kernel. Contour levels are shown in Figs. 5 and 6 for right ascension and declination respectively. In order to verify how good the different obtained models are, we study the mean and the variances of the random variables from the 


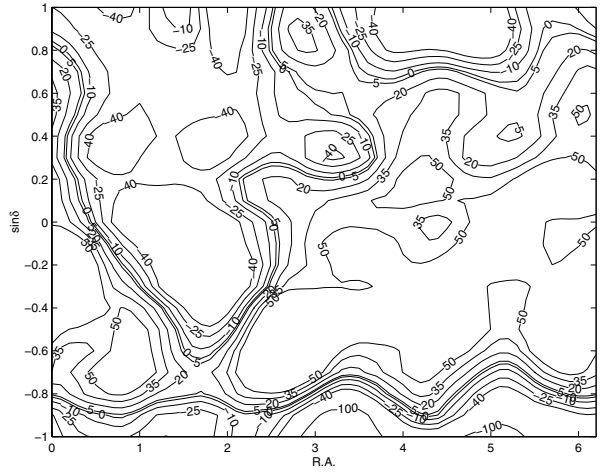

Fig. 5. Contour levels for $\Delta \alpha \cos \delta$ by means of the KNP model (in mas).

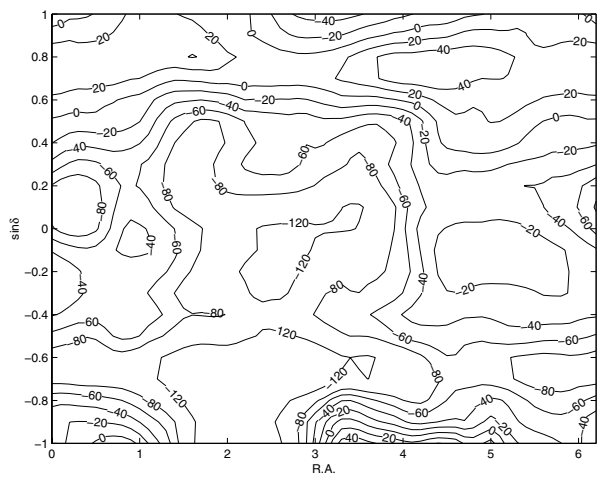

Fig. 6. Contour levels for $\Delta \delta$ by means of the KNP model (in mas).

net of points built for the regression (100 per 50 cells) To this end we apply the continuous definition. This requires numerical approximations of the integrals, and their comparison with the initial values and with those proposed by the restriction of the regression to the initial points.

It can be observed that the means are comparable for the initial points, for the regression with the kernel and for the values that this one induces for the initial points, while the standard deviations of the regression and the regression on the initial points decrease significantly, due to the occasional smoothness induced by the regression method (see Table 5). From this point, we study the values of the parameters that the nonparametric adjustment induces on the sphere for the GL, GLAD and GV models. This will serve as a contrast of the models in order to study an optimal strategy of adjustment. To this end, we take expression (9), where as $\Delta \alpha \cos \delta$ we take its nonparametric adjustment $m_{\alpha}(\alpha, \delta)$ and as $\Delta \delta$ the adjustment $m_{\delta}(\alpha, \delta)$. Thus, we can obtain the normal equations in a simple way and for any given model because the coefficients of the parameters to estimate are integrals which are easily computed, while the two independent terms may be computed by numerical integration from the evaluation of $m_{\alpha}(\alpha, \delta)$ and $m_{\delta}(\alpha, \delta)$ on discrete points. To preserve the orthogonality and also for convenience, we take these points equally spaced. Let us take $\phi_{x}=-\cos \alpha \sin \delta, \phi_{y}=-\sin \alpha \sin \delta, \phi_{z}=\cos \delta, \psi_{x}=$ $\sin \alpha, \psi_{y}=-\cos \alpha$ and 1 for the identically one function. Also, let $\langle\lambda, \zeta\rangle=\frac{1}{4 \pi} \int_{S^{2}} \lambda(\alpha, \delta) \zeta(\alpha, \delta) \cos \delta \mathrm{d} \alpha \mathrm{d} \delta$ be the usual scalar product for the real functions on the sphere and let us apply the condition of minimum to the GLAD, for example. We obtain the equations $A x=b$ where $x^{t}=\left[\Delta A, \epsilon_{x}, \epsilon_{y}, \epsilon_{z}, \Delta D\right]$ and:

$$
A=\left[\begin{array}{ccccc}
1 & 0 & 0 & \pi / 4 & 0 \\
0 & 2 / 3 & 0 & 0 & 0 \\
0 & 0 & 2 / 3 & 0 & 0 \\
\pi / 4 & 0 & 0 & 2 / 3 & 0 \\
0 & 0 & 0 & 0 & 1
\end{array}\right], b=\left[\begin{array}{c}
\langle\Delta \alpha \cos \delta, 1\rangle \\
\left\langle\Delta \alpha \cos \delta, \phi_{x}\right\rangle+\left\langle\Delta \delta, \psi_{x}\right\rangle \\
\left\langle\Delta \alpha \cos \delta, \phi_{y}\right\rangle+\left\langle\Delta \delta, \psi_{y}\right\rangle \\
\left\langle\Delta \alpha \cos \delta, \phi_{z}\right\rangle \\
\langle\Delta \delta, 1\rangle
\end{array}\right] .
$$

The corresponding equations for the GL model are, obviously, a particular case of these last equations. In Table 6 we present the values of the parameters in the GLAD and GL models, induced from the nonparametric adjustment according to the optimal values for the $h$ together with the means and variances of res $=(\Delta \alpha \cos \delta$ or $\Delta \delta)-$ Model that have the residuals after the adjustment with the two models, taking the initial stars. We also present the same statistical data for the residuals of the nonparametric adjustment (henceforth KNP).

It is interesting to note that the good behaviour of KNP for the 1327 reference stars is repeated over the complete sphere, where $E\left[K N P_{\alpha}\right]=10.0, \sqrt{\operatorname{Var}\left(K N P_{\alpha}\right)}=47.9$ and $E\left[K N P_{\delta}\right]=-48.5, \sqrt{\operatorname{Var}\left(K N P_{\delta}\right)}=51.8$. This data leads us to the following conclusions about KNP:

a) The model, applied over the sphere, has mathematical expectations for $\Delta \alpha \cos \delta$ and $\Delta \delta$ which to a large extent agree with the expectations of the random variables computed in Sect. 2 (see Table 1).

b) Equally, for the basis set of stars, there is an agreement between the sample mean and the mathematical expectations that show the coherence of the distribution of the initial points over the complete sphere.

c) The variances decrease as a consequence of the data smoothing process.

For these reasons the means and expectations of the residuals res $=$ Points - Model and res $=K N P-$ Model would be significant for the pruposes of our contrasts. In Tables 7 (basis point with sample means and standard deviations of the sample) and 8 (complete sphere with mathematical expectations and variances) these data appear for the MF, GL, GLAD direct (see Table 2) and GL, GLAD induced by regression (see Table 6) models.

The following conclusions can be drawn from this section:

1) The existence of a pure rotation between the FK5 and Hipparcos seems to be strongly implied by the stability of the coefficients $\epsilon_{x}$ and $\epsilon_{y}$ which were obtained by applying all the models. The value of $\Delta D$, when taken into account by the model, is also very stable.

2) On the contrary, uncertainties appear when we have to choose between $\epsilon_{z}$ and $\Delta A$ since they are strongly correlated. This will lead us to the possibility of using, as other authors have previously done, a mixed procedure which may consist of a GL or GLAD in addition to a KNP or a spherical harmonics adjustment. This question is studied below. A contrast for the $\epsilon_{z}$ value is presented in a later section.

3) However, after considering the results in Tables 7 and 8 it seems that, given that there is a bias in the initial sample 
Table 5. Means and standard deviations for the initial points, for the complete sphere and the discrete sample points after the smoothing process (in mas).

\begin{tabular}{lllllll}
\hline \hline & $\mu_{0}$ & $\mu_{S^{2}}$ & $\mu_{S^{2}}(1327$ stars $)$ & $\sigma_{0}$ & $\sigma_{S^{2}}$ & $\sigma_{S^{2}}(1327$ stars $)$ \\
\hline$\Delta \alpha \cos \delta$ & 11.4 & 10.0 & 12.7 & 83.46 & 47.98 & 45.12 \\
$\Delta \delta$ & -46.0 & -48.5 & -43.6 & 89.15 & 51.84 & 48.30 \\
\hline
\end{tabular}

Table 6. Means and standard deviations induced by the models in the initial subset of stars (in mas).

\begin{tabular}{llllllllll}
\hline \hline $\begin{array}{c}h_{\alpha}=0.55 \\
h_{\text {sin } \delta}=0.17\end{array}$ & \multirow{2}{*}{$\Delta A$} & $\Delta D$ & $\epsilon_{x}$ & $\epsilon_{y}$ & $\epsilon_{z}$ & $\mu\left(r_{\alpha}\right)$ & $\mu\left(r_{\delta}\right)$ & $\sigma\left(r_{\alpha}\right)$ & $\sigma\left(r_{\delta}\right)$ \\
\hline GLAD ind. & -39.1 & -48.5 & -19.4 & -13.9 & 62.5 & 0.0 & -2.0 & 82.4 & 86.7 \\
GL ind. & - & - & -19.4 & -15.9 & 16.4 & 0.6 & 2.0 & 82.5 & 86.1 \\
KNP & - & - & - & - & - & -1.31 & -2.4 & 63.40 & 69.4 \\
\hline
\end{tabular}

Table 7. Means and standard deviation for Points-model on the initial discrete points (in mas).

\begin{tabular}{llllll}
\hline \hline & Pts-KNP & Pts-GL dr & Pts-GLAD dr & Pts-GL ind. & Pts-GLAD ind. \\
\hline$\mu\left(r_{\alpha}\right)$ & -1.3 & -4.5 & 0.0 & -2.6 & 0.6 \\
$\sigma\left(r_{\alpha}\right)$ & 63.40 & 83.63 & 82.41 & 83.64 & 82.47 \\
$\mu\left(r_{\delta}\right)$ & -2.4 & -46.52 & -2.4 & -46.55 & 2.0 \\
$\sigma\left(r_{\delta}\right)$ & 69.44 & 86.05 & 69.44 & 85.96 & 86.07 \\
\hline
\end{tabular}

Table 8. Means and standard deviation for Points-model on the complete sphere (in mas).

\begin{tabular}{llllll}
\hline \hline & KNP-MF & KNP-GL dr & KNP-GLAD dr & KNP-GL ind. & KNP-GLAD ind. \\
\hline$E\left[r_{\alpha}\right]$ & -3.2 & 10.0 & -1.9 & -2.9 & 0.0 \\
$\operatorname{Var}\left(r_{\alpha}\right)$ & 48.14 & 40.06 & 45.52 & 48.47 & 45.79 \\
$E\left[r_{\delta}\right]$ & -48.5 & -48.5 & -1.7 & -48.5 & 0.0 \\
$\operatorname{Var}\left(r_{\delta}\right)$ & 46.54 & 46.41 & 46.28 & 46.24 & 46.38 \\
\hline
\end{tabular}

in $\Delta \alpha \cos \delta$ and also in $\Delta \delta$, the adoption of a model such as GLAD may be recommended.

\section{Spherical harmonics adjustment}

As we saw in the data analysis, the spatial distribution is practically homogeneous. This implies that the orthogonality of the spherical harmonics in the celestial sphere is well maintained in the discrete case from an algebraic point of view. The immediate consequence will be sufficient stability in the values of the coefficients of the developments when we increase their degree. In our case, we arranged the adjustments from degree 0 (where the arithmetical mean of the corresponding random variable should appear) to degree 5. In Tables 9 (for $\Delta \alpha \cos \delta$ ) and 10 (for $\Delta \delta$ ) we show the results for the different adjustments, although the coefficients up to order 2 are given.

From a statistical point of view, it should be stated that all the means of the residuals are null, while the variances are decreasing at the same time. Table 11 shows the standard deviation of the random variables according to the orders of the adjustments.
In spite of the decrease in the standard deviation, the increment of the order of the adjustment implies two difficulties consisting of the increment in the number of operations and, related to this, the decrease in the stability of the calculated coefficients. As a consequence, we maintain the order low, equal to or under five, and continue the analysis with them. These orders provide an acceptable stability for the coefficients, and it would therefore be interesting to see the values of the parameters that they induce for the GL, GV and GLAD models. More precisely, if $A d j$ represents a spherical harmonics adjustment (for $\Delta \alpha \cos \delta$ or $\Delta \delta$ and the order is any value between 0 and 5) and Mod is one of the models (GL, GLAD or GV) with parameters $\pi_{j}$, then for each star $i$ instead of working with the real values of $\Delta \alpha \cos \delta$ or $\Delta \delta$ in the condition equations, we can consider the following equations:

$\operatorname{Adj}\left(\alpha_{i}, \delta_{i}\right)=\operatorname{Mod}\left(\pi_{j}, \alpha_{i}, \delta_{i}\right) \quad$ for $\quad i=1,1327$.

Table 12 reflects the values obtained and shows how the values for each one of the different parameters correspond quite well with those obtained directly. Bearing in mind that these last values have been indirectly deduced from an independent 
Table 9. Coefficients for $\Delta \alpha \cos \delta$ development in spherical harmonics series (in mas).

\begin{tabular}{lllllll}
\hline \hline Coef $\backslash$ Ord & $n=0$ & $n=1$ & $n=2$ & $n=3$ & $n=4$ & $n=5$ \\
\hline 0,0 & 11.41 & 11.89 & 11.22 & 10.59 & 9.28 & 8.61 \\
$1,-1$ & - & 3.26 & 2.24 & 2.79 & 3.62 & 3.72 \\
1,0 & - & -23.77 & -20.83 & -17.04 & -14.45 & -13.15 \\
1,1 & - & -38.82 & -38.69 & -37.08 & -36.27 & -36.16 \\
$2,-2$ & - & - & 9.73 & 9.50 & 9.60 & 9.51 \\
$2,-1$ & - & - & 0.87 & 0.59 & 0.44 & 0.44 \\
2,0 & - & - & -37.20 & -41.78 & -47.03 & -49.03 \\
2,1 & - & - & -4.50 & -5.63 & -6.32 & -6.84 \\
2,2 & - & - & -1.78 & -1.39 & -1.37 & -1.47 \\
\hline
\end{tabular}

Table 10. Coefficients for $\Delta \delta$ development in spherical harmonics series (in mas).

\begin{tabular}{lllllll}
\hline \hline Coef $\backslash$ Ord & $n=0$ & $n=1$ & $n=2$ & $n=3$ & $n=4$ & $n=5$ \\
\hline 0,0 & -46.03 & -48.93 & -48.30 & -47.79 & -47.97 & -46.61 \\
$1,-1$ & - & 27.41 & 28.24 & 28.41 & 28.55 & 28.04 \\
1,0 & - & 58.49 & 56.19 & 55.16 & 54.96 & 49.45 \\
1,1 & - & -32.12 & -32.18 & -32.64 & -32.81 & -32.66 \\
$2,-2$ & - & - & -4.05 & -3.93 & -4.11 & -4.24 \\
$2,-1$ & - & - & -5.38 & -5.70 & -5.83 & -5.62 \\
2,0 & - & - & 29.51 & 31.22 & 30.81 & 38.77 \\
2,1 & - & - & 5.11 & 5.35 & 5.35 & 6.16 \\
2,2 & - & - & 1.81 & 1.79 & 1.73 & 1.73 \\
\hline
\end{tabular}

adjustment it is an acceptable verification for the accuracy of the parameters obtained.

We now carry out a statistical study, similar to that detailed in the previous section. Table 13 the references are the spherical harmonics (for the $\mathrm{SH}$, with $n=2,3$, or 4 representing the order, we refer to the initial points) or KNP (we refer to the sphere)

The following consequences are presented:

1) The means of the residuals between KNP and SHn (for $n=$ $2,3,4$ ) are null (evaluated in the complete sphere) and at the same time the standard deviation of the residuals are very similar to the deviations of the spherical harmonics with respect to the basis points in such a way that, with respect to the significance of the parameters, the models seem, in principle, to be adequate.

2) Nevertheless, if we observe the means and deviations of $\mathrm{SH}$ with respect to its induced models GL and GLAD, the first is biased in $\Delta \alpha \cos \delta$ and GLAD is practically unbiased for both variables and also has relatively lower deviations than those corresponding to KNP with the basis points, although they are comparable to them in the complete sphere.

\section{Two independent estimations for the $\epsilon_{\mathrm{z}}$ value}

The first main aim of this paper concerns the fact that the difference between our GLAD model and the GL, lies in the difference between their corresponding $\epsilon_{z}$ values. The main intrinsic difference between them rests on whether or not a value for $\Delta A$ is chosen. This is because there is a certain correlation between this value and $\epsilon_{z}$ because of the factor $\cos \delta$ that contains the latter: where $\cos \delta$ is the sum of the unity and an infinite development with $\sin ^{2} \delta$ as a variable. All the even order harmonics contribute something to the development, including the unity function itself. Thus, we need an independent method that gives us an idea of the true magnitude order of this parameter and to this end we can proceed in the following way: the KNP method consists of smoothing the data, which indicates that its calculation does not vary the rotations intrinsically contained in the initial data. In addition, we have spherical harmonics developments, also deduced from the initial data and exposed in Table 9 for the random variable $\Delta \alpha \cos \delta$. Where $\cos \delta$ is the $\epsilon_{z}$ coefficient, it is functionally dependent on the even harmonics and among these, the order 2 harmonic is independent of the coefficient of $\triangle A$ in the GLAD model, and it is also orthogonal to the coefficient functions of $\epsilon_{x}$ and $\epsilon_{y}$. Thus, it is particularly suitable to estimate the order of the magnitude $\epsilon_{z}$, which gives $\left(K N P, Y_{20}\right) \approx \epsilon_{z}\left(\cos \delta, Y_{20}\right)$. The calculations provide us an approximate value of 85.13 mas, which is in relatively strong agreement with those obtained with the (direct or induced) GLAD models.

Alternatively, it is possible to consider the harmonical development of order 3 and after obtaining the scalar product, $c_{20}\left(Y_{20}, Y_{20}\right) \approx \epsilon_{z}\left(\cos \delta, Y_{20}\right)$, with $c_{20}=31.22$. From this, we obtain an estimation of 63.62 mas, in this case which once more is better than the orders provided by the GLAD models. It is of interest to reiterate the independence of whether or not a value for $\Delta A$ is considered, which strengthens the viability of the best modelization of data obtained with the GLAD model induced by the optimal KNP.

\section{Mixed adjustments}

We now turn to our second goal; to decide what the best combination of an initial model could be, such as GL or GLAD, plus another complementary which may be a spherical harmonics adjustment (such as those proposed by Schwan) or a nonparametric adjustment by kernels to build a net of points with their associated values and proposing a table (in the same line as that recommended by Mignard \& Froeschle 2000). In the following subsections we deal with these questions more precisely.

\subsection{Basic model given by the surface spherical harmonics}

It is well known that in theory, the spherical harmonics developments may be obtained with as high a degree as desired. Hence, the variances decrease. In practice, the increase in the order implies, in addition to a large amount of calculations, an unavoidable increment in the instability of the coefficients. 
Table 11. Standard deviations for the different orders of the adjustments in spherical harmonics (in mas).

\begin{tabular}{lllllllllll}
\hline \hline$n$ & 2 & 3 & 4 & 5 & 6 & 7 & 8 & 9 & 10 & 15 \\
\hline$\sigma\left(\right.$ res $\left._{\alpha}\right)$ & 75.91 & 72.71 & 70.1 & 68.57 & 66.53 & 64.86 & 63.29 & 62.32 & 61.19 & 56.08 \\
$\sigma\left(\right.$ res $\left._{\delta}\right)$ & 77.57 & 77.07 & 76.18 & 72.56 & 71.6 & 70.59 & 69.34 & 67.98 & 66.95 & 62.36 \\
\hline
\end{tabular}

Table 12. Induced parameters for the different models from spherical harmonics developments (in mas).

\begin{tabular}{lccllllll}
\hline \hline & $\Delta A$ & $\Delta D$ & $\epsilon_{x}$ & $\epsilon_{y}$ & $\epsilon_{z}$ & $d_{1,0}$ & $d_{1,1}$ & $d_{1,-1}$ \\
\hline $\mathrm{GL}, n=2$ & - & - & -18.74 & -14.40 & 18.33 & - & - & - \\
$\mathrm{GLAD}, n=2$ & -52.07 & -46.53 & -19.45 & -14.74 & 79.44 & - & - & - \\
$\mathrm{GV}, n=2$ & - & - & -19.64 & -17.19 & 18.06 & -59.42 & -1.91 & 22.61 \\
$\mathrm{GL}, n=3$ & - & - & -18.95 & -14.72 & 18.33 & - & - & - \\
$\mathrm{GLAD}, n=3$ & -50.16 & -46.56 & -19.84 & -15.47 & 77.05 & - & - & - \\
$\mathrm{GV}, n=3$ & - & - & -19.85 & -17.52 & 18.06 & -59.38 & -1.84 & 22.71 \\
$\mathrm{GL}, n=4$ & - & - & -18.89 & -14.73 & 18.32 & - & - & - \\
$\mathrm{GLAD}, n=4$ & -57.82 & -46.55 & -19.51 & -15.37 & 86.85 & - & - & - \\
$\mathrm{GV}, n=4$ & - & - & -19.82 & -17.51 & 18.08 & -59.03 & -2.56 & 22.44 \\
$\mathrm{GL}, n=5$ & - & - & -18.57 & -15.11 & 18.33 & - & - & - \\
$\mathrm{GLAD}, n=5$ & -57.65 & -46.56 & -19.30 & -15.86 & 86.45 & - & - & - \\
$\mathrm{GV}, n=5$ & - & - & -19.48 & -17.91 & 18.07 & -59.26 & -2.30 & 22.79 \\
\hline
\end{tabular}

Table 13. Statistical values for the differences between KNP and different SHs (complete sphere) and SHs minus GL and GLAD inducted models (basis points, in mas).

\begin{tabular}{llllll}
\hline \hline & KNP-SH2 & KNP-SH3 & KNP-SH4 & SH2,3,4-GL ind. & SH2,3,4-GLAD ind. \\
\hline$\mu\left(r_{\alpha}\right)$ & -1.7 & -0.7 & 0.7 & 14.7 & 0.5 \\
$\sigma\left(r_{\alpha}\right)$ & 35.53 & 27.11 & 24.08 & $10.54,10.67,10.66$ & $32.53,39.31,43.05$ \\
$\mu\left(r_{\delta}\right)$ & 1.0 & 0.4 & 0.5 & 0.5 & -0.5 \\
$\sigma\left(r_{\delta}\right)$ & 29.04 & 27.63 & 26.31 & $16.58,16.83,16.81$ & $37.35,38.11,39.90$ \\
\hline
\end{tabular}

Although the distribution of points is close to uniform (see Sect. 2) it is clear that it is not so in a strict sense, and there is therefore a potential instability in the parameters of the development that is to be obtained. Taking into account that we want this distribution to be representative of the sample (which it is, since it deduces from it immediately) in the whole sphere, the zero order coefficients should approach the mean of the sample as closely as possible, in such a way that the estimator model has a very similar expectation to this mean. To clarify this simple question, all the parameters for each adjustment have been set free instead of increasing the order and maintaining the parameters with high reliability. As we saw in Tables 9 and 10, from order 4 on the variation of the main coefficient is notorious, and consequently an adjustment of order 3 was taken as a starting point. The values of the mean and standard deviation of the residuals over the basis points are given in Table 11 and to compute the corresponding expectation and standard deviation over the sphere, the adjustment KNP was used in auxiliary form, instead of the basis points, computed on the nodes of 100 per 50 cells. The results are presented in Table 13. The same table provides the statistical results for the differences, for the initial points, between this adjustment and the GL inducted and GLAD inducted models. We can see that GLAD is unbiased, while variances are lower for GL.

It is immediate to check that, for a given set of data, there is no difference between beginning with a GL and applying a spherical harmonics adjustment to the rest, or beginning with a GLAD and continuing with the spherical harmonics adjustment. Only the appearance of the expression and not the values of the final adjustment vary. Moreover, in both cases the means are null and the variances change with the order in the same way as indicated in Table 11 . We can conclude, in agreement with MF, that it is not possible to significantly deduce the standard deviations of the residuals by means of an increment in the order of the development. This does not mean that it is not of interest to obtain as good a development as possible. In all events, we must point out that when considering the functions over the sphere, the behaviour of both developments is different (we must not forget that in the discrete case, they are identical). The expectations are null for the GLAD and not for the GL. The variances are also lower for the GLAD. For example, for the right ascension it varies from 45.88 for the 3rd degree to 44.62 for the 15th, in the GL model, while in the GLAD model, it varies from 43.44 to 42.17 for the same orders. Therefore 
considered as a whole, it turns out that from a statistical point of view the adjustment obtained by summing up GLAD and the spherical harmonics for the differences is slightly better in the discrete and continuous case.

\subsection{Use of GLAD plus kernel nonparametric adjustment}

We have seen that the GLAD method is an unbiased method of minimum variance and it adapts perfectly with small variations, from a continuous point of view and also from a basis points point of view. The best complement for this method will be the KNP of the resulting residuals, with the optimal values for $h$ already used.

In particular, we have taken the GLAD model induced by the KNP found with the optimal values of the different $h$ for the initial differences. A new KNP was applied to the differences between these and the ones given by the application of the model, evaluated in such a way that 100 per 50 cells were built, with a value on each one of the nodes. From this, the following statistics were obtained for the resulting final residuals: for the complete sphere, the expectation for both random variable is -2.99 and 0 , while the standard deviations are 3.87 and 0.65 respectively. The statistics corresponding to the basic initial points are -0.4 and -0.92 for the arithmetical means and $12.07,12.91$ for the deviations. The final residuals are very close to the zero value. The results, taking as a basis the GL model, are similar or even better at the end, due to the fact that the model itself improves very slightly when compared with the initial errors (or its KNP adjustment) so in this case there is an improvement of the contribution of the model itself. It may be said that in the case of taking the GL model as a basis, almost all the correction is given by the nonparametric part. From this point of view, this slight statistical superiority (better expectation and final variance) is less interesting.

\section{Conclusions}

1) There is a bias in $\Delta \alpha \cos \delta$ and also in $\Delta \delta$. The former is more delicate to deal with.

2) The usually employed models search for infinitesimal rotations by means of the least squares method, but they do not remove the bias.

3) For any of the methods used, direct or indirect, there is a clear evidence of the existence of rotations. The $\epsilon_{x}, \epsilon_{y}$ rotations and the displacement $\Delta D$ are very stable. The existence of the bias in $\Delta \alpha \cos \delta$ makes the introduction of a $\Delta A$ coefficient in the adjustment cause a noticeable variation in the $\epsilon_{z}$ value.

4) While we are interested in a global adjustment for the whole sphere and we also want, as far as possible, this adjustment to be determined by certain parameters that will allow us to decide whether or not there is a pure rotation between the two catalogues.

5) The SH and KNP models do not make any supposition about dependence on right ascension and declination residuals. Therefore, we can use them in three ways: first, to see the GL and GLAD coefficient values that they imply; second, to decide on the $\epsilon_{z}$ value for two independent methods; third, to complete a basic GL or GLAD method in order to remove the remaining zonal errors.

6) All these difficulties led us to conduct a study that from the outset took into account the global point of view of the adjustment that we are prusuing. Thus, discussion of the models followed by the study of the mathematical methods and their compatibility with them, was required as a previous condition to finding the earlier conclusions.

7) The final conclusion on the most appropriate model to modelize Hipparcos-FK5 residuals is the GLAD model, not only for its statistical properties for the basis points, but also because these properties are extended to the whole sphere. However, a further possibility would be consider an $\epsilon_{z}$ value depending on zones in declination. This aim is beyond the scope of this work, in which we have prefered to focus on global values for the coefficients in the same vein as the papers of (Mignard \& Froeschlé 2000; Schwan 2001a)

8) As for the second goal of the paper, despite having mathematical security that bias and rotations between the systems exists, neither the models with rotations nor the models with rotations plus deformations seem enough to explain the zonal errors. A mixed model must be used which combines a basic one, such as GL or GLAD, and a KNP adjustment to build a net of points with their associated values to provide a numerical table.

Acknowledgements. We are very grateful to Dr. F. van Leeuwen for his suggestions and comments. We are also very grateful to Fundació Caixa Castelló BANCAIXA which has partially supported this paper.

\section{References}

Batrakov, Y. V., \& Chernetenko, Y. A. 1997, Journés 97, Systemes de reference Spatio-Temporels, ed. J. Vondrak, \& N. Capitaine (Prague), 22

Batrakov, Y. V., Chernetenko, Y. A., Gorel, G. K., \& Gudkova, L. A. 1999, A\&A, 352, 703

Branham, R. L., Jr. 1992, AJ, 103, 6

Branham, R. L., \& Sanguin, J. G. 1994, Proceedings of Third International Workshop on Positional Astronomy and Celestial mechanics (Valencia, Spain), 429

Bien, R., Fricke, W., Lederle, T., \& Schwan, H. 1978, Veröff. Astron. rechen-Inst (Heidelberg), No. 29

ESA 1997, The Hipparcos and Tycho Catalogues, SP-1200

Fricke, W., Scchwan, H., Lederle, T., et al. 1988, Veröff. Astron. rechen-Inst. Heidelberg, No. 32

Krasinsky, G. A., Pitjeva, E. V., et al. 2001, Russian Academy of Sciences, Institute of Applied Astronomy, 139

Hilton, J. L. 1996, Proceedings of Fourth International Workshop on Positional Astronomy and Celestial mechanics (Penyscola, Spain), 281

Lopez, J. A., Marco, F. J., \& Martinez, M. J. 1993, Proceedings of Second International Workshop on Positional Astronomy and Celestial mechanics (Valencia, Spain), 279

Mignard, F., Froeschlé, M., \& Turon, C. 1997, in The Hipparcos and Tycho Catalogues, SP-1200, 3, 415 
Mignard, F., \& Froeschlé, M. 2000, A\&A, 354, 732

Morse, P. M., \& Feshbach, H. 1953, Methods of Theoretical Phisics (McGraw-Hill)

Rapaport, M., \& Viateau, B. 1996, Proceedings of Fourth International Workshop on Positional Astronomy and Celestial mechanics (Penyscola, Spain), 237

Schwan, H. 2001, A\&A, 367, 1078

Schwan, H. 2001, A\&A, 373, 1099

Schwan, H. 2002, A\&A, 387, 1123

Simonoff, J. S. 1996, Smoothing Methods in Statistics (SpringerVerlag)

Shuygina, N. V., \& Yagudina, E. I. 1995, ed. S. Ferraz-Mello et al. (Paris, France), Proc. 172nd Symp. IAU

Viateau, B., Rapaport, M. 1995, A\&AS, 111, 305
Viateau, B., \& Rapaport, M. 1997, Journés 97, Systemes de reference Spactio-Temporels, ed. J. Vondrak, \& N. Capitaine (Prague), 130 Vityazev, V. V. 1997, Dynamics \& Astrometry of Natural and Artificial Celestial Bodies, 463

Vityazev, V. V., \& Yagudina, E. I. 1997, Journés 97, Systemes de reference Spactio-Temporels, ed. J. Vondrak, \& N. Capitaine (Prague), 153

Wand, M. P., \& Jones, M. C. 1995, Kernel Smoothing (Chapman \& Hall)

Yagudina, E. I. 1996, Proceedings of Fourth International Workshop on Positional Astronomy and Celestial mechanics (Penyscola, Spain), 59

Yagudina, E. I. 2001, Celestial Mechanics and dynamical Astronomy Zhu, Z., \& Yang, T. 1999, AJ, 117, 1103 